\title{
L5/S1Neuraxial space access
}

\author{
Charles H Ripp* \\ Jacob Ripp Student Georgetown University Washington DC, USA
}

\section{Introduction}

The difficulty in finding the epidural or spinal space for regional anesthesia required for surgical anesthesia or pain management may be profound. Understanding the time constraints and pressures of daily private practice led to our study of quick bedside access to the L5/S1 inter-laminar space for both pain management and surgical anesthesia/analgesia. It is well understood that scoliosis ends usually in the upper and mid lumbar areas in adults. The spine slowly degenerates in many adult patients evolving into a structure with facet arthosis, osteoarthritis, and collapse of disc spaces. These changes all cause narrowing and restriction for the anesthesia provider in an attempt to procure epidural or spinal access in pain management and required regional anesthesia for surgical patients. It is well known with modern fluoroscopic imaging that the L5/S1 space may be accessed efficientlyby $\mathrm{x}$-ray visualization even in the most degenerative spine. Therefore we felt a bedside non fluoroscopic technique for neuraxial access to the L5/ S1 inter-laminar space should be readily available for both bedside pain management and surgical treatment.

The authors are well aware of varying approaches and success using bony landmarks for gross accuracy (such as the iliac crest corresponding to the body of L5). Unfortunately using these gross bony landmarks sterility may be compromised, positioning may alter the bony landmarks relationship to the spine, and in the author's opinion under comparative fluoroscopic imaging, many of the prior techniques using bony landmarks have not led to a high consistent success of neuraxial access in the lower spine. We therefore undertook a simple study to access the ability of a $10 \mathrm{~cm}$ spinal needle on adults to accurately measure and be within under fluoroscopy the inter- laminar L5/S1 space from the superior border of the gluteal crease.

\section{Materials and methods}

100 adult patients in equal male and female numbers signed consents for the study. All patients were undergoing pain management procedures in the posterior spine andwere placed in prone position under fluoroscopy. An independent research RN recorded the measurements from the gluteal crease superior border to the interlaminar L5/S1 space under fluoroscopy. A recording of whether the needle was within or at the border of the inter-laminar space was also completed. All mid inter-laminar space length measurements (using a simple ruler) were taken after the $10 \mathrm{~cm}$ needle was placed from the superior border of the gluteal crease. We recorded as positive if the needle tip lay within the inter-laminar space or on the lamina border (superior or inferior) on direct PA fluoroscopic exam. The length of the distance from the superior border of the gluteal crease to the mid inter-laminar space was then as above recorded.

\section{Clinical research results}

95 of 100 patients had the $10 \mathrm{~cm}$ spinal needle at or within the lamina borders of L5/S1 under fluoroscopy. The measurements were roughly between 9 and $11 \mathrm{~cm}$. - with measurements to the .1 $\mathrm{cm}$ accuracy. The 5 measurements outside the lamina border were actually within $1 \mathrm{~cm}$ of being within the lamina under fluoroscopy. The difficulties encountered were pilonidal cyst repairs, obesity, muscle wasting, and asymmetric anatomy. The superior border of the gluteal crease is obvious in most adult patients. If the gluteal crease was not easily evident, we estimated where clinically we felt the crease occurred. All measurements were statistically significant with computerized $\mathrm{T}$ and $\mathrm{P}$ tests at $95 \%$ confidence intervals.

\section{Clinical conclusion}

The L5/5/S1 inter-laminar space is available for simple efficient neuraxial access with use of a simple $10 \mathrm{~cm}$. spinal needle. Measured from the superior border of the gluteal crease in adults, the L5/S1 neuraxial space is within or at the lamina border (superior or inferior) with $95 \%$ confidence. The lower lumbar neuraxial inter-laminar access at L5/S1 for spinal or epidural regional anesthesia was able to be revealed under fluoroscopy efficiently in a very high percentage of patients with the use of a simple $10 \mathrm{~cm}$. spinal needle guide.

\section{Discussion}

The author has performed an immense number of regional anesthetics in pain management and OR anesthesia for 30 years. I've witnessed and experienced personally the difficulty with patients modernly with the demands of required sedation for regional anesthesia, obesity, neuraxial degeneration, positioning, and limited time and required successful precise treatment. This led to the study since I had used this technique in both the sitting and prone positions for many years when neuraxial access was difficult. Obviously the L5/S1 inter-laminar space used for spinal and epidural anesthesia is not optimal for many procedures. Despite this when difficulty with neuraxial access in upper lumbar areas and time constraints occur, this method has been a superb technique. Utilizing baricity and positioning with subarachnoid anesthesia and epidural catheter threading (or combination treatment), virtually all the occurrences when surgical anesthesia or post op analgesia to the mid-thoracic dermatomes

Correspondence to: Ripp $\mathrm{CH}, \mathrm{MD}$, Private Anesthesia Practice Colorado Springs Co, Jacob Ripp Student Georgetown University Washington DC, USA, Tel: 719-648-7253, Fax: 719-592-0020; E-mail: hrmdco@comcast.net

Key words: anesthesia, neuraxial, pain management

Received: October 29, 2015; Accepted: November 16, 2015; Published: November 20, 2015 
was needed, this method worked well. I have used this method for C-Sections in obese patients, post op analgesia for total hips and knees, abdominal surgery as a combined technique leading to post op neuraxialanalgesiaand for simple spinal/epidural narcotic treatments needed from the thoracic areas to the lower extremities with high personal success.

This method is not perfect, and many of my surgical and pain patients are sitting or in the lateral decubitus position for specific neuraxial treatment. The study was performed with prone patients. I do feel the correlation with varying position is high with this simple technique - due to fixed anatomic structures as landmarks. I don't feel however without fluoroscopic or ultrasound that the technique is perfect. Patient and needle movements may cause deviation from an intended needle path. The L5 spinous process contact with midline or paramedian approaches occurs not uncommonly. This quick bedside technique however has saved me on countless occasions from repeated needle attempts on both awake and sedated patients when upper lumbar access was difficult. I consider this an adjunctive technique when one is frustrated with the upper lumbar approach, under time constraints, or has a patient that cannot tolerate repeated attempts. Obviously depending on the needed dermatomal level for analgesia/anesthesia, appropriate rapid positioning, appropriate required volume, baricity and speed of drug administered, and catheter threading length are crucial to the success of the needed analgesia/anesthesia.

Copyright: @2015 Ripp CH. This is an open-access article distributed under the terms of the Creative Commons Attribution License, which permits unrestricted use, distribution, and reproduction in any medium, provided the original author and source are credited. 